\title{
Medical microbiology, infectious diseases, and the public health: a trio in search of harmony
}

\author{
B I Duerden
}

There is no doubt that infection is still a major part of medical practice. It was never perceived to be otherwise outside the "developed" world of Europe and North America, and after a period of culpable complacency the medical profession and public alike now realise that infections are still, and will, remain high on the medical agenda. Far from going away, problems of infection are increasing, albeit often of a different nature.

Various branches within the medical and allied professions are concerned with infection. The three main groups of doctors whose primary role concerns infections are medical microbiologists and virologists, infectious diseases physicians, and public health medicine doctors, exemplified specifically now by the consultants for communicable disease control (CCDC). Over many years the three branches developed independently in terms of professional affilitation and training. They must now face the problem of co-ordinating and integrating their activities to provide the services necessary for the investigation, treatment, and prevention of infection.

In the 1960 s and early 1970 s there was a serious decline in interest in infection that affected all three branches: "antibiotics had controlled bacterial infection" and "vaccines were eliminating epidemic infectious diseases." Few young doctors entered medical microbiology or virology, the number of infectious diseases physicians declined, and the medical officer of health $(\mathrm{MOH})$ became the medical officer for environmental health (MOEH) with a wide range of responsibilities of which infection was only one component, and in the view of many, a very minor component. This complacency received several sharp jolts in the late 1970s and 1980s with the discovery of new pathogens (Campylobacter, Legionella, etc), the recognition that salmonella food poisoning and nosocomial infection were affecting tens of thousands of patients annually in the United Kingdom, and above all, in the view of the media, politicians, and the public, the advent of AIDS.

The irony that it was two hospital outbreaks of infection (salmonella food poisoning at Stanley Royd Hospital and legionellosis at Stafford) which brought about the Acheson Report on the public health ${ }^{1}$ has been highlighted by O'Brien $e t a l^{2}$ This resulted in the creation of CCDC responsible for infection in the community. Before then, medical microbiologists had found their services in much greater demand from their clinical colleagues, and had responded, in particular to the need for expertise in hospital infection control. The role of infectious diseases physicians was also changing from traditional "fevers" to a wider range of modern infective problems.

As they adapt to meet the challenges of infective problems, the three branches should not be regarded, nor regard themselves, as competing factions, but as complementary and essential links in a single, cohesive service. One important aspect of integration is mutual acceptance of the related training programmes; up to now these have been quite separate, but if the services are to come closer together, training must inevitably become more integrated and overlapping. Changes in medical postgraduate training that will come from the Report of the Calman Committee ${ }^{3}$ offer the opportunity to define training elements which are appropriate to all infection specialties and will provide greater flexibility for trainees.

The Royal Colleges of Pathologists and Physicians and the Faculty of Public Health Medicine have been concerned about the differences and barriers among the three groups. There have been several attempts through joint working parties and liaison committees to draw them together. Sadly, a joint RCPath/RCP committee in the mid-1980s foundered when it produced a document on joint training ${ }^{4}$ that caused disharmony between microbiologists and infectious diseases physicians and was out of date before it appeared.

More successful was the Joint Working Party on CCDC Training established in 1989. It has produced outline training programmes accessible to trainees who started their careers in public health medicine, microbiology, or clinical infectious diseases. ${ }^{5}$ Having fulfilled its remit, that committee dissolved itself in May 1993, handing on responsibility for this particular area to a new Intercollegiate Standing Committee on Infection, formed by the two Royal Colleges and the Faculty, with a much wider remit encompassing all aspects of infection services. This committee should begin its work in mid1994 and will need (i) to define the types of 
services and the professional roles needed for the future and (ii) to devise interrelated training programmes with sufficient flexibility. There have been some calls for a Faculty of Microbial Diseases, distinct from the current professional bodies, but I do not believe that most members of each branch would find this attractive. Microbiologists have an essential laboratory base that links them with their other colleagues in pathology; infectious diseases physicians have clinical responsibilities that overlap with other branches of internal medicine; and CCDCs need to maintain links with the broader areas of public health medicine, particularly for those who enter the specialty through that initial route.

In an integrated infection service, which could appropriately be considered as a Department of Microbial Diseases, as proposed by the Association of Medical Microbiologists in their publication on the "undervalued asset", 6 there should be three core elements, integrated professionally and each a consultant-led clinical activity: a microbiology/virology laboratory service; a clinical service comprising inpatient care, outpatient clinics, and clinical consultation, and advice on individual patients and on general policies; and an epidemiology and community service. Many professional colleagues other than the three key groups contribute to these services to a greater or lesser extent, including other clinicians (in genitourinary medicine, paediatrics, clinical immunology, respiratory medicine, oncology, etc), nurses, clinical scientists and medical laboratory scientific officers, and epidemiologists, but each provides only a part of the service needed.

The number of people in such a service and their jobs within the system will vary from place to place, depending on the size, scale, and range of the service provided. The essential elements required of an infection service in any district or NHS Trust hospital are laboratory investigations, clinical advice, clinical care of patients, hospital infection control activities, antibiotic and other policies, community preventive measures and epidemiological data gathering. Who provides these elements?

Laboratory investigations are provided by the microbiology/virology laboratory headed by a consultant microbiologist/virologist trained to determine what tests should be done, supervise the laboratories, interpret the results and advise colleagues.

Clinical advice is provided by medical microbiologists/virologists or infectious diseases physicians, in somewhat different circumstances. As the microbiology services have developed in the United Kingdom over the past 30 years, most clinical advice on infection is provided by medical microbiologists/virologists. This situation is unlikely to change and should not change. This is not only because all except 12 districts in England and Wales have at least one consultant microbiologist whereas infectious dis- eases physicians are concentrated in specialist, often regional, units, but because most such advice arises from the microbiologist recognising clinical problems by monitoring the results in the laboratory and from regular ward visits and attendance at ward rounds on key clinical units. Only a minority of such items of advice are initiated by a specific request from a clinician; the reverse is often true of requests for consultation and advice from infectious diseases physicians. Where microbiologists and infectious diseases physicians are closely linked and operate as an integrated team, the "unsolicited" advice element tends to be more evenly split between them.

Clinical care In regional centres specialist inpatient and outpatient care is provided by infectious diseases physicians, elsewhere by other physicians, often with a particular interest and expertise in some aspect of infection. Microbiologists rarely have direct clinical responsibility for inpatients, but in some places they contribute to specific outpatient clinics, such as sexually transmitted diseases and urinary tract infection.

Hospital infection control is usually the responsibility of a consultant microbiologist who is also infection control doctor and is contracted to commit, on average, two to three sessions a week to these activities. ${ }^{7}$ In a few hospitals this role is taken by an infectious diseases physician. The CCDC has a co-ordinating role when hospital infection problems impinge on the community or in the production of more general district-wide policies involving more than one unit.

Antibiotic and other policies in relation to infection are generally initiated and co-ordinated by the consultant microbiologists because of their pivotal role in monitoring patterns of sensitivity through laboratory testing and providing advice on antibiotic treatment. In hospitals or districts with an infectious diseases unit, such policies are generally the result of collaboration between microbiologists and infectious diseases physicians. However, to be effective, the infection specialists must involve their other clinical colleagues to achieve a consensus based on scientific evidence-imposition by dictat has little chance of success.

Community preventive measures and co-ordination are the specific responsibility of the CCDC, with the support and co-operation of the medical microbiologist and infectious diseases physicians. Such integration is often achieved best when the CCDC has a close physical link with the microbiology department.

From this assessment of duties, two patterns of staffing emerge as appropriate for an infection service.

(1) In a regional or academic centre with sufficient specialist clinical demand all three components will be needed and should form an integrated and interactive service-consultant medical microbiologists/ virologists, infectious diseases physicians, and a full-time district CCDC. 
(2) In a smaller district or unit direct and personal involvement of a consultant microbiologist will be provided in the laboratory serving that unit-either on site or close enough to provide that personal input. Specialist infectious diseases consultation will be available through the regional centre. The CCDC may, in many cases, be part-time, sharing duties as CCDC for another district, or with other duties in public health medicine, or as a part-time microbiologist. In all of these cases professional support and cover are often provided by the medical microbiologist.

If this integrated approach is to be achieved, present consultants in the three branches need to come together to co-ordinate their service, and, perhaps more importantly, trainees must be provided with appropriate training programmes. Most of the elements are already there: with enthusiasm and goodwill from all concerned, it can be achieved.

1 The Committee of Inquiry into the Future Development of the Public Health Function. (Acheson Report) Public Health in England. London: HMSO, 1988.

2 O'Brien JM, O'Brien SJ, Geddes AM, Heap BJ, MayonWhite RT. Tempting fate-control of communicable disease in England. BMF 1993;306:1461-4.

3 Working Group on Specialist Medical Training. Hospital doctors-Training for the future. London: HMSO, 1993.

4 Joint Working Party of the Royal College of Physicians and the Royal College of Pathologists. Training in infectious diseases. London: 1989. Royal College

5 Duerden BI, Miller DL. Training in Communicable Disease Control. Bull Roy Coll Pathol 1993;83:7-8.

6 Medical microbiology and the control of infections. Medical microbiology for the NHS and community. (1) $A n$ undervalued asset. London: Media Medica, 1988.

7 Duerden BI, Roberts C, Desselberger U. Joint planning of training grade numbers in medical microbiology and virology: submission from the Royal College of virology: submission from the Royal College of Laboratory Service for presentation to the committee. Bull Roy Coll Pathol 1993;83:xii-xix. 